GANIT J. Bangladesh Math. Soc. (ISSN 1606-3694) 39 (2019) 45-62

DOI: https://doi.org/10.3329/ganit.v39i0.44165

\title{
IMPACT OF TREATMENT ON HIV-MALARIA CO- INFECTION BASED ON MATHEMATICAL MODELING
}

\author{
Amit Kumar Saha ${ }^{1}$, Ashrafi Meher Niger ${ }^{2}$, Chandra Nath Podder ${ }^{3 *}$ \\ ${ }^{1,2,3}$ Department of Mathematics, Dhaka University, Dhaka-1000, Bangladesh \\ "Corresponding author: cpodder@du.ac.bd
}

Received: 03-03-2019

Accepted: 11-11-2019

\begin{abstract}
The distribution of HIV and malaria overlap globally. So there is always a chance of co-infection. In this paper the impact of medication on HIV-Malaria co-infection has been analyzed and we have developed a mathematical model using the idea of the models of Mukandavire, et al. [13] and Barley, et al. [3] where treatment classes are included. The disease-free equilibrium (DFE) of the HIV-only model is globally-asymptotically stable (GAS) when the reproduction number is less than one. But it is shown that in the malaria-only model, there is a coexistence of stable disease-free equilibrium and stable endemic equilibrium, for a certain interval of the reproduction number less than unity. This indicates the existence of backward bifurcation. Numerical simulations of the full model are performed to determine the impact of treatment strategies. It is shown that malaria-only treatment strategy reduces more new cases of the mixed infection than the HIV-only treatment strategy. Moreover, mixed treatment strategy reduces the least number of new cases compared to single treatment strategies.
\end{abstract}

Keywords: HIV, Malaria, Co-infection, Treatment

\section{Introduction}

In the Sub-Saharan region of Africa, malaria and HIV are endemic. There are many factors behind the high incidence of HIV in Sub-Saharan Africa and one of the key factors is the dual infection with malaria and the geographic overlap between these two diseases [1, 14]. According to the World Health Organization (WHO), Malaria and HIV are responsible for more than two million deaths each year [23]. According to the Center for Disease Control and Prevention (CDC), about $5 \%$ increase occurs in malaria related deaths due to co-infection with HIV [7]. From these results we can guess that biological differences could alter the effect of co-infection. It is found that death rates due to malaria have almost doubled for co-infections with HIV [1].

After the discovery of human immune-deficiency virus (HIV), the intermediary of the acquired immune deficiency syndrome (AIDS) in 1981, it has become a new threat to global health and human development [17]. It has killed more than 25 million people worldwide but the HIV

(C) GANIT: Journal of Bangladesh Mathematical Society, 2019 
prevalent in Bangladesh is still considerable [1, 15]. As a result of infection with the virus, the immune system deteriorates dynamically. HIV infection increases poverty, reduces the workforce and poses tremendous pressure on limited health and social services [19, 22]. On the other hand, malaria is a vector-borne disease. According to the World Malaria Report 2016, in 2015, almost 429000 people died from malaria globally. It is a threat to the unprivileged and it causes impoverishment [2]. If malaria is untreated, it attacks the liver and moves through the blood stream infecting every organ leading to death [3]. HIV promotes the chance of malaria infection and increases the development of symptoms of malaria in persons whose immune-system is very weak. The main purpose is to evaluate the consequence of treatment for HIV and malaria [4]. For this we shall formulate HIV-malaria co-infections model with treatment class $[13,17]$.

HIV infection is often associated with several comorbidities caused by the presence of malaria [21]. Studies show that co-infection fuels each other and information on co-infection effect on immune system is important for patient care [10, 18].

The paper is decorated as follows. In section 2, a basic HIV-Malaria co-infection model, with treatment as an intervention strategy, is formulated and analyzed. HIV-only and malaria-only sub models are exploredin sections 3 and 4. Then the full model is explored in section 5. Simulation works areperformed in details in section 6.

\section{Model Formulation and Basic Properties}

The whole human demography $N(t)$, at time $t$, is sub-divided into the following sub-populations: being susceptible to all pathogens $S(t)$,being infected with malaria only $I_{M}(t)$, being infected with HIV only $I_{H}(t)$, and being infected with both HIV and malaria $I_{H M}(t)$. Moreover, proportion of treated individuals infected with HIV only is represented by $W_{H}(t)$, proportion of treated individuals infected with malaria only is represented by $W_{M}(t)$, and proportion of treated individuals infected with both HIV and malaria is represented by $W_{H M}(t)$. So the total human populations are given by

$$
N(t)=S(t)+I_{H}(t)+I_{M}(t)+I_{H M}(t)+W_{H}(t)+W_{M}(t)+W_{H M}(t) .
$$

Again the wholemosquito population $N_{V}(t)$, at time t, is subdivided into susceptible $S_{V}(t)$ and infectious $I_{V}(t)$. The total vector population is

$$
N_{V}(t)=S_{V}(t)+I_{V}(t)
$$

\section{Assumptions}

Here we consider some assumptions. These are followings:

A1: The newly sexually active susceptible individuals $(S)$ are recruited into the human population at a constant rate $\Lambda$.

A2: The susceptible vector populations $\left(S_{V}\right)$ are generated at a constant $\Lambda_{V}$. 
A3: The natural death rate for human is $\alpha$ and for vector is $\mu_{V}$. The disease induced death rate for HIV-infected individuals is $\mu_{H}$, for malaria-infected individuals is $\mu_{M}$ and for mixed HIV-malaria infected individuals is $\mu_{H M}$.

A4: The rate at which malaria infected individuals recover from malaria and return to the susceptible class is $(\vartheta)$. Individuals with mixed HIV-malaria infection recover from malaria and may return to the HIV class at a reduced rate. Here the scaling parameter $k$ is introduced for the recovery rate of malaria from mixed HIV-malaria infection and after recovery from malaria, individuals return to the HIV class at a rate $(k \vartheta)$.

A5: Susceptible vector populations acquire infection only from infected individuals and susceptible humans acquire infection only from infected mosquitoes.

A6: Individuals successfully treated for HIV are assumed to eventually succumb to the disease (due to the failure of treatment or resistance development) and progress to HIV at a reduced rate $\sigma$, where $0<\sigma<1$. Again, individuals in this class progress to malaria at a reduced rate $\sigma_{M}$, where $0<\sigma_{M}<1$. Individuals successfully treated for mixed HIV-malaria are assumed to eventually attack with the HIV disease (due to the failure of treatment or resistance development) and progress to HIV at a reduced rate $\sigma_{1}$, where $0<\sigma_{1}<1$.

Now we discuss about the transmission of the diseases.

\section{Transmission by singly-infected individuals:}

Susceptible individuals get infected with HIV at a rate $\lambda_{H}$, given by

$$
\lambda_{H}=\frac{\beta_{H}\left(I_{H}+\eta_{H} W_{H}\right)}{N} .
$$

Similarly, susceptible individuals get infected with malaria at a rate $\lambda_{M}$, which is given by

$$
\lambda_{M}=\frac{\beta_{M} I_{V} b_{M}}{N} .
$$

\section{Transmission by Dually-Infected individuals:}

\section{Untreated Individuals:}

Untreated dually-infected individuals (those in the $I_{H M}$ class) transmit HIV at a rate

$$
\lambda_{M H 1}=\frac{\beta_{H} \rho_{1} I_{H M}}{N} .
$$

\section{Treated Individuals:}

Treated individuals with the mixed infection transmit HIV at a rate $\lambda_{M H 2}$, where

$$
\lambda_{M H 2}=\frac{\beta_{H} \eta_{H} W_{H M}}{N} .
$$

Adding all the three terms $\left(\lambda_{H}, \lambda_{M H 1}, \lambda_{M H 2}\right)$ and expressing it as $\lambda_{H 1}$, we get

$$
\lambda_{H 1}=\lambda_{H}+\lambda_{M H 1}+\lambda_{M H 2} .
$$


Mosquitoes acquire malaria parasite at force of infection rate $\lambda_{V}$, where $\lambda_{V}$ is given by

$$
\lambda_{V}=\frac{\beta_{M V} b_{M}\left(I_{M}+\rho_{2} I_{H M}\right)}{N} .
$$

Considering all suppositions we have the underlying system of differential equations:

$$
\begin{aligned}
& S^{\prime}=\Lambda+v I_{M}+\rho W_{M}-\lambda_{H 1} S-\lambda_{M} S-\alpha S, \\
& I_{M}{ }^{\prime}=\lambda_{M} S-\delta \lambda_{H 1} I_{M}-k_{1} I_{M}, \\
& I_{H}{ }^{\prime}=\lambda_{H 1} S+v k I_{H M}+\sigma W_{H}+\sigma_{1} W_{H M}-\theta \lambda_{M} I_{H}-k_{2} I_{H} \\
& I_{H M}{ }^{\prime}=\delta \lambda_{H 1} I_{M}+\theta \lambda_{M} I_{H}+\sigma_{M} W_{H}-k_{3} I_{H M}, \\
& W_{M}{ }^{\prime}=\tau_{1} I_{M}-k_{4} W_{M}, \\
& W_{H}{ }^{\prime}=\tau_{2} I_{H}-k_{5} W_{H}, \\
& W_{H M}{ }^{\prime}=\tau I_{H M}-k_{6} W_{H M}, \\
& S_{V}{ }^{\prime}=\Lambda_{V}-\lambda_{V} S_{V}-\mu_{V} S_{V}, \\
& I_{V}{ }^{\prime}=\lambda_{V} S_{V}-\mu_{V} I_{V},
\end{aligned}
$$

where, prime denotes derivative and

$$
\begin{aligned}
& k_{1}=v+\alpha+\tau_{1}+\mu_{M}, k_{2}=\alpha+\tau_{2}+\mu_{H}, \\
& k_{3}=\alpha+\tau+\mu_{H M}+v k, \\
& k_{4}=\rho+\alpha, k_{5}=\alpha+\sigma_{M}+\sigma, k_{6}=\alpha+\sigma_{1} .
\end{aligned}
$$

The model flow diagram is delineated in Fig.1.and the parameters are described in Table 1.

Table 1. Description of parameters for the model (6).

\begin{tabular}{|c|l|}
\hline Parameter & \multicolumn{1}{|c|}{ Description } \\
\hline$\Lambda, \Lambda_{V}$ & Recruitment rate of humans and mosquitoes \\
\hline$\beta_{H}$ & Effective contact rate for HIV infection \\
\hline$\beta_{M}, \beta_{M V}$ & Transmission probability for malaria in humans and vectors \\
\hline$\eta_{H}, \eta_{M}$ & modification parameter \\
\hline$b_{M}$ & biting rate of mosquitoes \\
\hline$\mu_{H}, \mu_{M}, \mu_{H M}$ & Disease induced mortality for HIV, malaria and mixed infection \\
\hline$\alpha, \mu_{V}$ & Natural death rate of humans and mosquitoes \\
\hline$\rho$ & Progression rate to susceptible class of individuals treated for malaria \\
\hline$\rho_{1}, \rho_{2}$ & Modifications parameters \\
\hline$\tau_{1}, \tau_{2}, \tau$ & Treatment rates for $I_{H}, I_{M}$ and $I_{H M}$ classes \\
\hline$\sigma, \sigma_{M}$ & Progression rate to HIV or malaria by HIV infected treated individuals \\
\hline$\sigma_{1}$ & Progression rate to HIV by dually infected treated individuals \\
\hline$v$ & Recovery rate of humans from malaria \\
\hline$k$ & Reduction factor of the recovery rate for HIV-Malaria co-infection \\
\hline
\end{tabular}




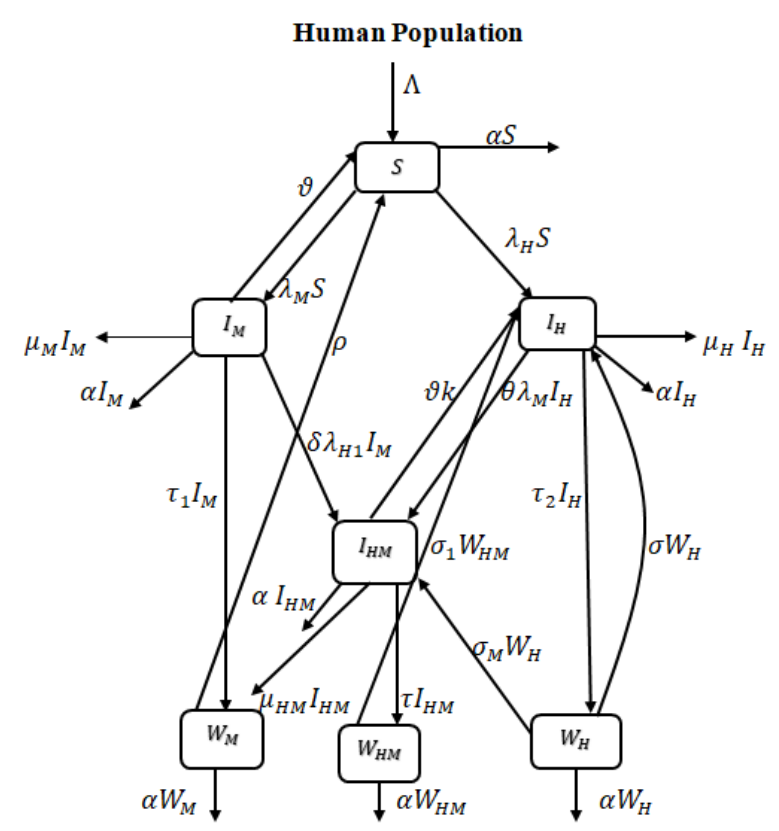

\section{Mosquito Population}

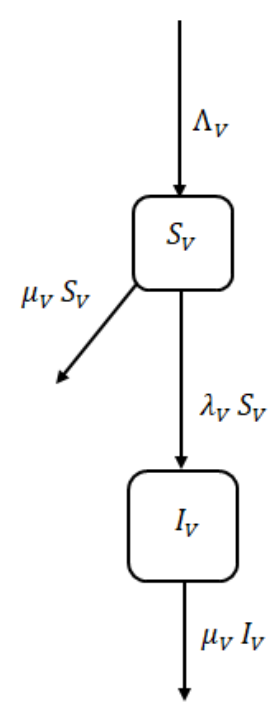

Fig. 1: Schematic diagram of the HIV-Malaria model (6)

\section{HIV-only Model}

The HIV-only model is

$$
\begin{aligned}
& S^{\prime}=\Lambda-\lambda_{H} S-\alpha S, \\
& I_{H}{ }^{\prime}=\lambda_{H} S+\sigma W_{H}-k_{2} I_{H}, \\
& W_{H}{ }^{\prime}=\tau_{2} I_{H}-(\alpha+\sigma) W_{H},
\end{aligned}
$$

where, $\lambda_{H}=\frac{\beta_{H}\left(I_{H}+\eta_{H} W_{H}\right)}{N}$, and $N=S+I_{H}+W_{H}$.

For this model, it can be shown that the region $\Omega_{H}=\left\{\left(S, I_{H}, W_{H}\right) \in \mathbb{R}_{+}{ }^{3}: N \leq \frac{\Lambda}{\alpha}\right\}$ is positively invariant and attracting with respect to the system $(7)[13,16,17]$.

\section{Local Stability of DFE}

The DFE, $\varepsilon_{H 0}$, of the HIV-only model (7), is

$$
\varepsilon_{H 0}=\left(S^{*}, I_{H}{ }^{*}, W_{H}^{*}\right)=\left(\frac{\Lambda}{\alpha}, 0,0\right) \text {. }
$$

For the system (7), calculating the matrices $F$ and $V$, for the new infection terms and the remaining transfer terms [20], it can be shown thatthe basic reproduction number [9], denoted by $\mathcal{R}_{H}$, is given by

$$
\mathcal{R}_{H}=\rho\left(F V^{-1}\right)=\frac{\beta_{H}\left(\sigma+\alpha+\eta_{H} \tau_{2}\right)}{k_{2}(\sigma+\alpha)-\sigma \tau_{2}},
$$


where, $\rho$ represents the spectral radius of $F V^{-1}$. The following result is established using Theorem 2 [20].

Lemma1. The DFE of the HIV-only model (7), given by (8), is locally-asymptotically stable (LAS) if $\mathcal{R}_{H}<1$, and unstable if $\mathcal{R}_{H}>1$.

The following results can be established (using the techniques in [11, 17])

Theorem 1. The DFE of the HIV-only model (7), given by $\varepsilon_{H 0}$, is GAS whenever $\mathcal{R}_{H}<1$.

Proof. The following Lyapunov function is considered:

$$
\mathcal{F}=f_{1} I_{H}+f_{2} W_{H},
$$

where, $f_{1}=\frac{\sigma+\alpha+\eta_{H} \tau_{2}}{\eta_{H} k_{2}+\sigma}, \quad$ and $f_{2}=1$.

with Lyapunov derivative (where a dot denotes differentiation with respect to $t$ ).

$$
\text { So } \begin{aligned}
\dot{\mathcal{F}} & =f_{1} \dot{I}_{H}+f_{2} \dot{W}_{H}, \\
& =\left(\sigma+\alpha+\eta_{H} \tau_{2}\right) \dot{I}_{H}+\left(\eta_{H} k_{2}+\sigma\right) \dot{W}_{H}, \\
& =\left(\sigma+\alpha+\eta_{H} \tau_{2}\right)\left(\lambda_{H} S+\sigma W_{H}-k_{2} I_{H}\right)+\left(\eta_{H} k_{2}+\sigma\right)\left(\tau_{2} I_{H}-(\alpha+\sigma) W_{H}\right), \\
& =\left(\sigma+\alpha+\eta_{H} \tau_{2}\right) \lambda_{H} S-\left(I_{H}+\eta_{H} W_{H}\right)\left(\alpha k_{2}+\sigma k_{2}-\sigma \tau_{2}\right), \\
& =\left(\sigma+\alpha+\eta_{H} \tau_{2}\right) \lambda_{H} S-\frac{\left(\alpha k_{2}+\sigma k_{2}-\sigma \tau_{2}\right) N}{\beta_{H}} \lambda_{H}, \\
& =\frac{\left(\sigma k_{2}+\alpha k_{2}-\sigma \tau_{2}\right) \lambda_{H} N}{\beta_{H}}\left(\frac{\beta_{H}\left(\sigma+\alpha+\eta_{H} \tau_{2}\right) S}{\left(k_{2}(\sigma+\alpha)-\sigma \tau_{2}\right) N}-1\right), \\
& \leq \frac{\left(\sigma k_{2}+\alpha k_{2}-\sigma \tau_{2}\right) \lambda_{H} N}{\beta_{H}}\left(\mathcal{R}_{H}-1\right),(\text { since } S \leq N), \\
& \leq 0 \text { for } \mathcal{R}_{H} \leq 1 .
\end{aligned}
$$

Since all the parameters are non-negative, $\dot{\mathcal{F}} \leq 0$ for $\mathcal{R}_{H} \leq 1$ with $\dot{\mathcal{F}}=0$ if and only if $I_{H}=$ $W_{H}=0$. Hence $\mathcal{F}$ is a Lyapunov function on $\Omega_{H}$. Therefore, by the LaSalle's invariance principle [11], every solution to the equations of the model (7), with initial conditions in $\Omega_{H}$, approaches $\varepsilon_{H 0}$ as $t \rightarrow \infty$, whenever $\mathcal{R}_{H} \leq 1$.

From the above results we can say that HIV will be eliminated if $\mathcal{R}_{H}$ can be brought to a value less than unity.

\section{Existence of Endemic Equilibrium (EE)}

Let EE point be denoted by $\varepsilon_{1}=\left(S^{*}, I_{H}{ }^{*}, W_{H}{ }^{*}\right)$,

$$
\text { and } \lambda_{H}{ }^{*}=\frac{\beta_{H}\left(I_{H}{ }^{*}+\eta_{H} W_{H}{ }^{*}\right)}{S^{*}+I_{H}{ }^{*}+W_{H}{ }^{*}} \text {. }
$$

Setting the right hand sides of the model to zero (and noting that $\lambda_{H}{ }^{*}=\lambda_{H}$ at equilibrium) gives 


$$
\begin{aligned}
S^{*} & =\frac{\Lambda}{\alpha+\lambda_{H}{ }^{*},} \\
I_{H}{ }^{*} & =\frac{(\alpha+\sigma) \Lambda \lambda_{H}{ }^{*}}{\left(\alpha+\lambda_{H}{ }^{*}\right)\left(\alpha k_{2}+\sigma\left(\alpha+\mu_{H}\right)\right)^{\prime}} \\
W_{H}{ }^{*} & =\frac{\tau_{2} \Lambda \lambda_{H}{ }^{*}}{\left(\alpha+\lambda_{H}{ }^{*}\right)\left(\alpha k_{2}+\sigma\left(\alpha+\mu_{H}\right)\right)} .
\end{aligned}
$$

Using (11) in the expression for $\lambda_{H}{ }^{*}$ in (10) shows that the nonzero (endemic) equilibria of the model satisfy

$$
a_{11} \lambda_{H}{ }^{*}-a_{12}=0
$$

where, $\quad a_{11}=\frac{\alpha+\sigma+\tau_{2}}{\alpha\left(k_{2}+\sigma+\mu_{H}\right)}$, and $\quad a_{12}=\mathcal{R}_{H}-1$.

If $\mathcal{R}_{H}>1$ then $a_{12}>0$, and $a_{11}$ is always positive. Thus, from the linear system (12) $\lambda_{H}{ }^{*}=a_{12} /$ $a_{11}$, which is unique and positive whenever $\mathcal{R}_{H}>1$. Then substituting $\lambda_{H}{ }^{*}=a_{12} / a_{11}$ into (11) we can determine the components of the endemic equilibrium $\varepsilon_{1}$. Again, if $\mathcal{R}_{H}<1$ then $a_{12}<0$ and hence $\lambda_{H}{ }^{*}<0$ (which does not mean anything biologically). Hence there is no positive equilibria. These can be abbreviated below.

Lemma 2. The HIV-only model (7) has a unique EE if and only if $\mathcal{R}_{H}>1$, and no EE otherwise.

\section{Local Stability of Endemic Equilibrium}

To study the local asymptotic stability of the endemic equilibrium the center manifold theory $[5,6]$ is used. To do this, the following simplification and change of variables are made. Let $S=$ $x_{1}, I_{H}=x_{2}, W_{H}=x_{3}$ so that

$N=x_{1}+x_{2}+x_{3}$. Moreover, by using the vector notation $=\left(x_{1}, x_{2}, x_{3}\right)^{T}$, the HIV-only model (7) can be written in the form $\frac{d X}{d t}=\left(f_{1}, f_{2}, f_{3}\right)^{T}$, as follows:

$$
\begin{aligned}
& \frac{d x_{1}}{d t}=f_{1}=\Lambda-\frac{\beta_{H}\left(x_{2}+x_{3} \eta_{H}\right) x_{1}}{x_{1}+x_{2}+x_{3}}-\alpha x_{1}, \\
& \frac{d x_{2}}{d t}=f_{2}=\frac{\beta_{H}\left(x_{2}+x_{3} \eta_{H}\right) x_{1}}{x_{1}+x_{2}+x_{3}}-\left(\alpha+\mu_{H}+\tau_{2}\right) x_{2}+\sigma x_{3}, \\
& \frac{d x_{3}}{d t}=f_{3}=\tau_{2} x_{2}-\alpha x_{3}-\sigma x_{3} .
\end{aligned}
$$

The Jacobian of the system (13) at $\varepsilon_{H 0}$, is given by

$$
J\left(\varepsilon_{H 0}\right)=\left(\begin{array}{ccc}
-\alpha & -\beta_{H} & -\beta_{H} \eta_{H} \\
0 & -\left(\alpha+\mu_{H}+\tau_{2}\right)+\beta_{H} & \sigma+\beta_{H} \eta_{H} \\
0 & \tau_{2} & -(\alpha+\sigma)
\end{array}\right) .
$$


When $\mathcal{R}_{H}=1$, let $\beta_{H}=\beta^{*}$ be a bifurcation parameter. From (9) using $\mathcal{R}_{H}=1$ gives the expression for $\beta_{H}$ as

$$
\beta_{H}=\beta^{*}=\frac{(\alpha+\sigma) k_{2}-\sigma \tau_{2}}{\left(\alpha+\sigma+\eta_{H} \tau_{2}\right)} .
$$

Here the Jacobian $\left(J\left(\varepsilon_{H 0}\right)\right)$ of (13) at the DFE has one eigenvalue of magnitude zero and the remaining eigenvalues have negative real part. So, the idea of center manifold theory $[5,6]$ can be applied to show the LAS of the EEP of (13) and hence of the original system (7), for $\beta_{H}$ near $\beta^{*}$.

Eigenvectors of $\mathrm{J}_{\beta^{*}}=\left.\mathrm{J}\left(\varepsilon_{\mathrm{H} 0}\right)\right|_{\beta_{\mathrm{H}}=\beta^{*}}$ :

For $\mathcal{R}_{H}=1$, the jacobian of (13) at $\beta_{H}=\beta^{*}$ denoted by $\left(J_{\beta^{*}}\right)$ has a right eigenvector associated with the zero eigenvalue which is given by $=\left[w_{1}, w_{2}, w_{3}\right]^{T}$, where,

$$
w_{1}=-\frac{\left(\beta^{*} w_{2}+\beta^{*} \eta_{H} w_{3}\right)}{\alpha}, w_{2}=w_{2}, w_{3}=\frac{\tau_{2} w_{2}}{\alpha+\sigma} .
$$

Moreover, $J_{\beta^{*}}$ has a left eigenvector $v=\left[v_{1}, v_{2}, v_{3}\right]$, where,

$$
v_{1}=0, v_{2}=v_{2}, v_{3}=\frac{\left(\sigma+\beta^{*} \eta_{H}\right) v_{2}}{\alpha+\sigma} .
$$

\section{Computations of a and $b$ :}

For the system (13), to determine the sign of $a$

$$
\text { we have, } a=\sum_{k, i, j=1}^{n} v_{k} w_{i} w_{j} \frac{\partial^{2} f_{k}}{\partial x_{i} \partial x_{j}}(0,0) \text {, }
$$

Using the associated non-zero partial derivatives of $F$ (at the DFE) and after some algebraic manipulations it can be easily shown that the expression for $a$ is

$$
a=-\frac{2 v_{2} \beta^{*} \alpha\left(w_{2}+w_{3}\right)\left(w_{2}+\eta_{H} w_{3}\right)}{\Lambda}<0 .
$$

For the sign of $b$ we have

$$
b=\sum_{k, i=1}^{n} v_{k} w_{i} \frac{\partial^{2} f_{k}}{\partial x_{i} \partial \varnothing}(0,0) .
$$

Using the associated non-zero partial derivatives of $F$ (at the DFE) and after some algebraic manipulations it can be easily shown that the expression for $b$ is

$$
b=v_{2}\left(w_{2}+w_{3} \eta_{H}\right)>0 .
$$

Thus $a<0$ and $b>0$. So (by Center Manifold theorem [5, 6]) the following result can be established (the result holds for $\mathcal{R}_{H}>1$ but close to 1 ). 
Theorem 2. The unique endemic equilibrium confirmed by Center Manifold theory [5, 6] is LAS for $\mathcal{R}_{H}$ near 1 . In brief, the DFE of the HIV-only model (7) is GAS whenever $\mathcal{R}_{H} \leq 1$, and it has a unique endemic equilibrium point whenever $\mathcal{R}_{H}>1$.

\section{Malaria-only Model}

The malaria-only model is given by:

$$
\begin{aligned}
& S^{\prime}=\Lambda+v I_{M}+\rho W_{M}-\lambda_{M} S-\alpha S, \\
& I_{M}{ }^{\prime}=\lambda_{M} S-k_{1} I_{M}, \\
& W_{M}{ }^{\prime}=\tau_{1} I_{M}-k_{4} W_{M}, \\
& S_{V}{ }^{\prime}=\Lambda_{V}-\lambda_{V} S_{V}-\mu_{V} S_{V}, \\
& I_{V}{ }^{\prime}=\lambda_{V} S_{V}-\mu_{V} I_{V},
\end{aligned}
$$

where, $\lambda_{M}=\frac{\beta_{M} B_{M} I_{V}}{N}, \lambda_{V}=\frac{\beta_{M V} B_{M} I_{M}}{N}$, and $N=S+I_{M}+W_{M}$.

\section{Local stability of the DFE}

The DFE $\left(\varepsilon_{M 0}\right)$ of the malaria-only model (15) is given by

$$
\varepsilon_{M 0}=\left(S, I_{M}, W_{M}, S_{V}, I_{V}\right)=\left(\frac{\Lambda}{\alpha}, 0,0, \frac{\Lambda_{V}}{\mu_{V}}, 0\right) .
$$

The basic reproduction number, denoted by $\mathcal{R}_{M}$, is

$$
\mathcal{R}_{M}=\frac{b_{M} \sqrt{\Lambda \Lambda_{V} \beta_{M} \beta_{M V} \alpha k_{1}}}{\Lambda \mu_{V} k_{1}} .
$$

Thus, using Theorem 2 [20] the following result can be established.

Theorem3. The DFE of the malaria-only model (15) is LAS if $\mathcal{R}_{M}<1$, and unstable if $\mathcal{R}_{M}>1$.

\section{The Endemic Equilibrium (EE):}

Let the EEP of the system (15) be given by

$\varepsilon_{M 1}=\left(S^{*}, I_{M}{ }^{*}, W_{M}{ }^{*}, S_{v}{ }^{*}, I_{v}{ }^{*}\right)$, and $\left(\lambda_{M}{ }^{*}=\lambda_{M}\right.$ at equilibrium $)$

So, $\lambda_{M}{ }^{*}=\frac{\beta_{M} b_{M} I_{V}^{*}}{N^{*}}, \lambda_{V}{ }^{*}=\frac{\beta_{M V} b_{M} I_{M}{ }^{*}}{N^{*}}$, and $N^{*}=S^{*}+I_{M}{ }^{*}+W_{M}{ }^{*}$.

Setting the right hand sides of the model to zero we solve for $S^{*}, I_{M}{ }^{*}, W_{M}{ }^{*}, S_{v}{ }^{*}, I_{v}{ }^{*}$, and using these expressions along with $\lambda_{V}{ }^{*}$ in $\lambda_{M}{ }^{*}$ we get the following polynomial equation (in terms of $\left.\lambda_{M}{ }^{*}\right)$

$$
\lambda_{M}{ }^{*}\left[A\left(\lambda_{M}{ }^{*}\right)^{2}+B \lambda_{M}{ }^{*}+C\right]=0 .
$$

where, $A=\mu_{V} \Lambda\left(\tau_{1}+k_{4}\right)\left(\beta_{M V} b_{M} k_{4} \Lambda+\mu_{V} k_{4} \Lambda+\mu_{V} \Lambda \tau_{1}\right)$, 


$$
\begin{aligned}
B= & \left(\beta_{M V} b_{M} k_{4} \Lambda+\mu_{V} k_{4} \Lambda+\mu_{V} \Lambda \tau_{1}+\mu_{V} k_{1} k_{4} \Lambda^{2}\left(\tau_{1}+k_{4}\right)\right) \mu_{V} \\
& -\beta_{M V} \beta_{M} b_{M}{ }^{2} k_{4} \Lambda \Lambda_{V}\left(\alpha k_{4}+\mu_{M} k_{4}+\alpha \tau_{1}\right), \\
C= & \mu_{V}{ }^{2}{k_{1}}^{2} k_{4}{ }^{2} \Lambda^{2}\left(1-\mathcal{R}_{m}{ }^{2}\right) .
\end{aligned}
$$

Equation (17) says that the non-zero equilibria of the model satisfy

$$
f\left(\lambda_{M}{ }^{*}\right)=A\left(\lambda_{M}{ }^{*}\right)^{2}+B \lambda_{M}{ }^{*}+C=0 .
$$

Hence, we have the following result.

Theorem 4. The model (15) has

(i) exactly an identical $\mathrm{EE}$ if $C<0$ (i.e., $\mathcal{R}_{M}>1$ ),

(ii) exactly an identical EE if $B<0$, and $C=0$ or $B^{2}-4 A C=0$,

(iii) exactly two $\mathrm{EE}$ if $B<0, C>0$ (i.e., $\mathcal{R}_{M}<1$ ) and $B^{2}-4 A C>0$,

(iv) no EE otherwise.

\section{Existence of Backward Bifurcation}

From the above Theorem 3 we see that the DFE of the malaria-only model is LAS if $\mathcal{R}_{M}<1$. Still, it may not assure the global asymptotic stability of the equilibrium for $\mathcal{R}_{M}<1$, which indicates the possibility of backward bifurcation $[8,9,16]$.This is investigated below, using the center manifold theorem [5,6]. Let $S=x_{1}, I_{M}=x_{2}, W_{M}=x_{3}, S_{V}=x_{4}, I_{V}=x_{5}$.

The Jacobian of the system (15) at the DFE $\varepsilon_{M 0}$ is denoted by $J\left(\varepsilon_{M 0}\right)$ and is given by :

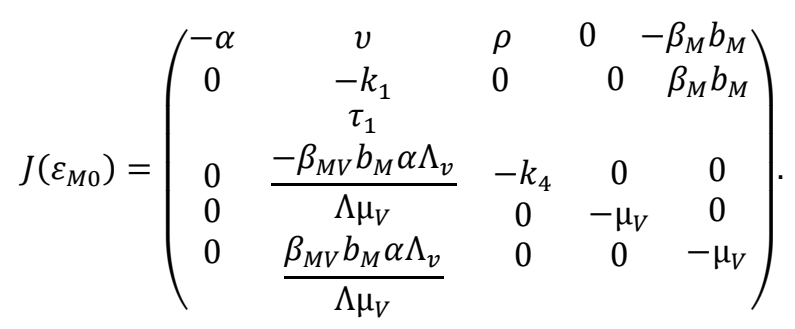

When $\mathcal{R}_{M}=1$, let $\beta_{M}=\beta^{*}$ be a bifurcation parameter. Solving (16) for $b_{M}$ from $\mathcal{R}_{M}=1$ gives

$$
\beta_{M}=\beta^{*}=\frac{\mu_{V}^{2} k_{1}^{2} \alpha^{2}}{\alpha \beta_{M V} b_{M}{ }^{2} k_{1} \Lambda \Lambda_{V}} .
$$

Eigenvectors of $\mathrm{J}_{\beta^{*}}=\left.\mathbf{J}\left(\varepsilon_{\mathrm{M} 0}\right)\right|_{\beta_{\mathrm{M}}=\boldsymbol{\beta}^{*}}$ :

When $\mathcal{R}_{M}=1$, the Jacobian at $\beta_{M}=\beta^{*}$ expressed by $\left(J_{\beta^{*}}\right)$ has a right eigenvector

$$
w=\left[w_{1}, w_{2}, w_{3}, w_{4}, w_{5}\right]^{T},
$$

where, $w_{1}=\frac{v w_{2}+\rho w_{3}-b_{M} \beta^{*} w_{5}}{\alpha}, w_{2}=w_{2}, w_{3}=\frac{\tau_{1} w_{2}}{k_{4}}, w_{4}=\frac{-\beta_{M V} b_{M} \alpha \Lambda_{v} w_{2}}{\Lambda \mu_{V}{ }^{2}}$ 
and $\quad w_{5}=\frac{\beta_{M V} b_{M} \alpha \Lambda_{v} w_{2}}{\Lambda \mu_{V}^{2}}$.

Moreover, $J_{\beta^{*}}$ has a left eigenvector

$$
\begin{aligned}
& v=\left[v_{1}, v_{2}, v_{3}, v_{4}, v_{5}\right], \text { where, } \\
& v_{1}=0, v_{2}=v_{2}, v_{3}=0, \\
& v_{4}=0, v_{5}=\frac{\beta^{*} b_{M} v_{2}}{\mu_{V}}
\end{aligned}
$$

\section{Computations of a and $b$ :}

After some calculation the expression for $a$ is $a=-\frac{2 b_{M} \alpha \beta_{M V} v_{5} w_{2} w_{4}(\Delta-1)}{\Lambda}$, where,

$$
\Delta=\frac{\Lambda \beta^{*} \mu_{V w_{2} w_{5}}\left(w_{2}+w_{3}\right)+\alpha \Lambda_{V} \beta_{M V} v_{5} w_{2}\left(w_{1}+w_{2}+w_{3}\right)}{\Lambda \beta_{M V} \mu_{V} v_{5} w_{2 w_{4}}},
$$

andthe expression for $b$ is

$$
b=v_{2} w_{5} b_{M}>0 .
$$

The sign of $a$ is not known. Hence, it follows (from Center Manifold Theorem [5, 6]) that model (15) experiences backward bifurcation at $\mathcal{R}_{M}=1$ whenever

$$
a=-\frac{2 b_{M} \alpha \beta_{M V} v_{5} w_{2} w_{4}(\Delta-1)}{\Lambda}>0 .
$$

This is abbreviated below.

Theorem 5. The malaria-only model (15) experiences backward bifurcation at $\mathcal{R}_{M}=1$ whenever inequality (20) is satisfied.

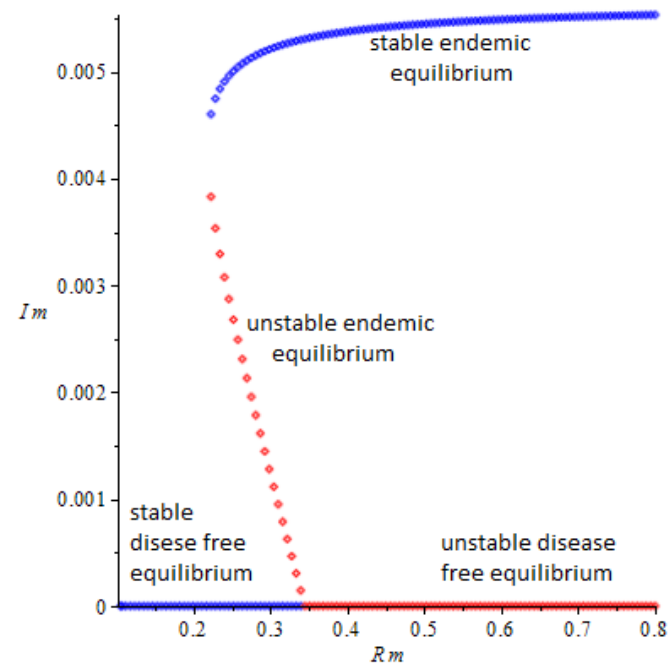

Figure 2.This figure demonstrates the occurrence of backward bifurcation for the malaria only model (15) using: $\Lambda=0.001, \Lambda_{V}=0.01, \alpha=0.015, \mu_{M}=0.15, \mu_{V}=0.06, \rho=0.06, \beta_{M V}=0.08, v=0.5$, $\tau_{1}=0.07, b_{M}=0.8$ and different values of $\beta_{M}$, we have $a>0$ and $b>0$. 
It is illustrated in (Figs. 2-3.). Using parameter values, we have $\mathcal{R}_{M}=0.6737$ and $a=$ 0.00007681434774 with $b=0.03525$. The following figure (Fig. 2.) has been generated using Maple to illustrate the phenomenon of backward bifurcation for the malaria only model (15).

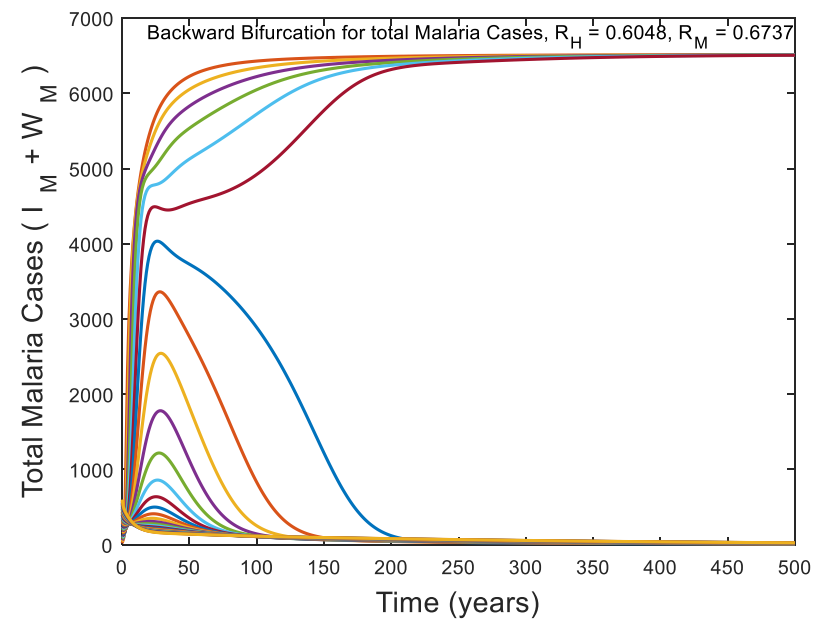

Figure 3. This figure demonstrates the occurrence of backward bifurcation for malaria-only with different initial conditions in the model (6). Parameter values used are as in Table 2 with $\mu_{H}=0.01, \mu_{V}=0.75, \rho=$ $0.1, v=1.5, k=1, \tau=5, \tau_{1}=0.4, \tau_{2}=4, \sigma=0.0303, \sigma_{M}=0.02, \sigma_{1}=0.033, b_{M}=1$.

\section{Analysis of the HIV-Malaria Model}

\section{Local Stability of the DFE:}

DFE of (6) is given by

$$
\begin{aligned}
\varepsilon_{0} & =\left(S, I_{M}, I_{H}, I_{H M}, W_{M}, W_{H}, W_{H M}, S_{V}, I_{V}\right), \\
& =\left(\frac{\Lambda}{\alpha}, 0,0,0,0,0,0, \frac{\Lambda_{V}}{\mu_{V}}, 0\right) .
\end{aligned}
$$

The reproduction number for thefull model (6) (denoted by $\mathcal{R}_{H M}$ ) is

$$
\mathcal{R}_{H M}=\max \left\{\mathcal{R}_{H}, \mathcal{R}_{M}\right\} .
$$

Using Theorem 2 [20], we have the following result.

Theorem6.The DFE of the model (6) is LAS if $\mathcal{R}_{H M}<1$, and unstable if $\mathcal{R}_{H M}>1$.

Model (6) also experiences backward bifurcation as the malaria-only model (15). We claim the following.

Theorem 7. The full model (6) experiences backward bifurcation at $\mathcal{R}_{H M}=1$ whenever inequality (23) is satisfied.

Proof. This can also be proved using the center manifold theorem [5, 6] on the model (6). 
let $S=x_{1}, I_{M}=x_{2}, I_{H}=x_{3}, I_{H M}=x_{4}$ and $W_{M}=x_{5}, W_{H}=x_{6}, W_{H M}=x_{7}, S_{V}=x_{8}, I_{V}=$ $x_{9}$.

The Jacobian of the system (6) at the DFE point $\varepsilon_{0}$, is denoted by $J\left(\varepsilon_{0}\right)$ and is given by

$$
J\left(\varepsilon_{0}\right)=\left(\begin{array}{cccccccccc}
-\alpha & v & -\beta_{H} & -J_{1} & \rho & -J_{2} & -J_{2} & 0 & -J_{3} \\
0 & -k_{1} & 0 & 0 & 0 & 0 & 0 & 0 & J_{3} \\
0 & 0 & J_{4} & J_{5} & 0 & J_{6} & J_{7} & 0 & 0 \\
0 & 0 & 0 & -k_{3} & 0 & \sigma_{M} & 0 & 0 & 0 \\
0 & \tau_{1} & 0 & 0 & -k_{4} & 0 & 0 & 0 & 0 \\
0 & 0 & \tau_{2} & 0 & 0 & -k_{5} & 0 & 0 & 0 \\
0 & 0 & 0 & \tau & 0 & 0 & -k_{6} & 0 & 0 \\
0 & -J_{8} & 0 & -J_{9} & 0 & 0 & 0 & -\mu_{V} & 0 \\
0 & J_{8} & 0 & J_{9} & 0 & 0 & 0 & 0 & -\mu_{V}
\end{array}\right) .
$$

where, $J_{1}=\beta_{H} \rho_{1}, J_{2}=\beta_{H} \eta_{H}, J_{3}=\beta_{H} b_{M}, J_{4}=-k_{2}+\beta_{H}, J_{5}=\beta_{H} \rho_{1}+v k, J_{6}=\beta_{H} \eta_{H}+\sigma$,

$$
J_{7}=\beta_{H} \eta_{H}+\sigma_{1}, \quad J_{8}=\frac{\beta_{M V} b_{M} \alpha \Lambda_{V}}{\Lambda \mu_{V}}, J_{9}=\frac{\beta_{M V} b_{M} \alpha \Lambda_{V} \rho_{2}}{\Lambda \mu_{V}} .
$$

And $\mathcal{R}_{H M}=\max \left\{\mathcal{R}_{H}, \mathcal{R}_{M}\right\}$ as before,

where, $\mathcal{R}_{H}=\frac{\beta_{H}\left(\sigma+\alpha+\eta_{H} \tau_{2}\right)}{k_{2}(\sigma+\alpha)-\sigma \tau_{2}}$, and $\mathcal{R}_{M}=\frac{\sqrt{\Lambda \Lambda_{V} \beta_{M} \beta_{M V} \alpha k_{1}}}{\Lambda \mu_{V} k_{1}}$.

When $\mathcal{R}_{H M}=1$ (that is, $\mathcal{R}_{H}<\mathcal{R}_{M}=1$ ), let $\beta_{M}=\beta^{*}$ be a bifurcation parameter.

Then $\mathcal{R}_{M}=1$ gives the expression for $\beta_{M}$ as $\beta_{M}=\beta^{*}=\frac{k_{1}^{2} \alpha^{2} \mu_{V}^{2}}{\Lambda \Lambda_{V} \alpha k_{1} \beta_{M V} b_{M}^{2}}$.

Eigenvectors of $\mathbf{J}_{\boldsymbol{\beta}^{*}}=\left.\mathbf{J}\left(\boldsymbol{\varepsilon}_{\mathbf{0}}\right)\right|_{\boldsymbol{\beta}_{\mathbf{M}}=\boldsymbol{\beta}^{*}}$ :

when $\mathcal{R}_{H M}=1$, the Jacobian of (6) at $\beta_{M}=\beta^{*}$ expressed by $\left(J_{\beta^{*}}\right)$ has a right eigenvector

$w=\left[w_{1}, w_{2}, w_{3}, w_{4}, w_{5}, w_{6}, w_{7}, w_{8}, w_{9}\right]^{T}$, where,

$w_{1}=\frac{v w_{2}-\beta_{H} w_{3}-J_{1} w_{4}+\rho w_{5}-J_{2} w_{6}-J_{2} w_{7}-J_{3} w_{9}}{\alpha}$,

$w_{2}=w_{2}, w_{3}=\frac{k_{5} w_{6}}{\tau_{2}}, w_{4}=w_{4}, w_{5}=\frac{\tau_{1} w_{2}}{k_{4}}$,

$w_{6}=w_{6}, w_{7}=\frac{\tau w_{4}}{k_{6}}, w_{8}=-\frac{j_{8} w_{2}+J_{9} w_{4}}{\mu_{V}}, w_{9}=\frac{k_{1} w_{2}}{J_{3}}$.

Further, $J_{\beta^{*}}$ has a left eigenvector $v=\left[v_{1}, v_{2}, v_{3}, v_{4}, v_{5}, v_{6}, v_{7}, v_{8}, v_{9}\right]$, where,

$v_{1}=0, v_{2}=v_{2}, v_{3}=-\frac{\tau_{2} w_{6}}{J_{4}}, v_{4}=\frac{J_{5} v_{3}+\tau v_{7}+J_{9} v_{9}}{k_{3}}, v_{5}=0, v_{6}=v_{6}, v_{7}=\frac{J_{7} v_{3}}{k_{7}}, v_{8}=0, v_{9}=\frac{k_{1} v_{2}}{J_{8}}$.

\section{Computations of $a$ and $b$ :}

After some calculations, it can be shown that

$$
b=b_{M} v_{2} w_{9}>0
$$

Thus, it follows from Center Manifold Theorem [5, 6] that the model (6) experiences backward 
bifurcation at $\mathcal{R}_{H M}=1$ whenever

$$
a>0 \text {. }
$$

This is illustrated in Fig.4.

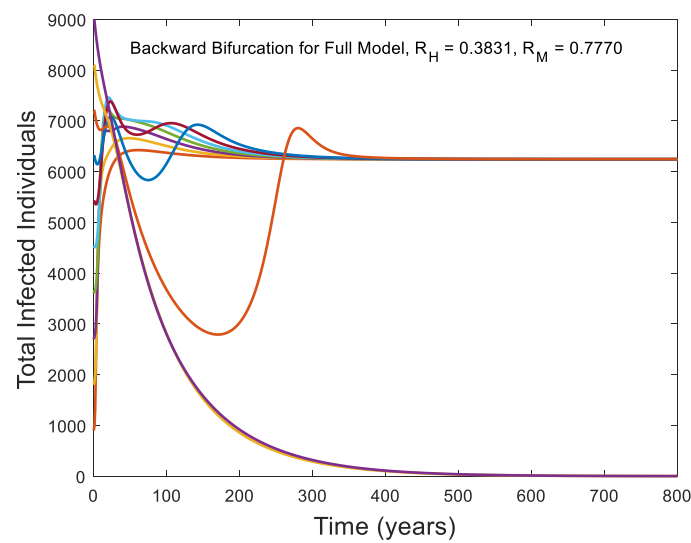

Fig. 4. This figure demonstrates the occurrence of backward bifurcation for the full model with different initial conditions in the model (6). Parameter values are obtained from Table 2 with $\alpha=0.02, \mu_{H}=0.01$, $\mu_{V}=0.75, \mu_{H M}=0.1, \beta_{H}=0.6, v=1.5, \tau=5, \tau_{1}=0.4, \tau_{2}=5, \sigma=0.04, \sigma_{M}=0.04, \sigma_{1}=0.05$, $b_{M}=1$.

Table 2. Description of parameters for the model (6).

\begin{tabular}{|c|c|c|}
\hline Parameter & Nominal value & References \\
\hline$\Lambda, \Lambda_{V}$ & 100,100000 & {$[12]$} \\
\hline$\beta_{H}, \beta_{M}, \beta_{M V}$ & $0.75,0.6,0.6$ & {$[12]$} \\
\hline$\eta_{H}, \sigma, \sigma_{M}, \sigma_{1}$ & $0.001,0.003,0.006,0.001$ & assumed \\
\hline$b_{M}$ & $(0.25,1)$ & {$[12]$} \\
\hline$\mu_{H}, \mu_{M}, \mu_{H M}, \mu_{V}$ & $0.02,0.2,0.01,6$ & {$[12]$} \\
\hline$\alpha, \rho, \rho_{1}, \rho_{2}$ & $0.015,0.1,1.02,1.002$ & assumed \\
\hline$\tau_{1}, \tau_{2}, \tau$ & Variable(treatment rate) & {$[17]$} \\
\hline$v, \delta, \theta, k$ & $0.005,1.002,1.00,0.5$ & {$[12]$} \\
\hline
\end{tabular}

\section{Evaluation of treatment strategies}

The full model (6) is now simulated to describe the treatment strategies as follows:

\section{HIV-only treatment strategy}

Here individuals in the $I_{H}$ and $I_{H M}$ class are treated at a rate $\tau_{2}$. In this case $\tau_{1}=0$ and $\tau=0$. Using $\tau_{2}=0.02$, the results, illustrated in Fig.5a and Fig.5b, show that the number of new cases of HIV-infection and the mixed HIV-Malaria infection have reduced significantly. The result follows the similar trends for higher treatment rate (Fig.5.). 


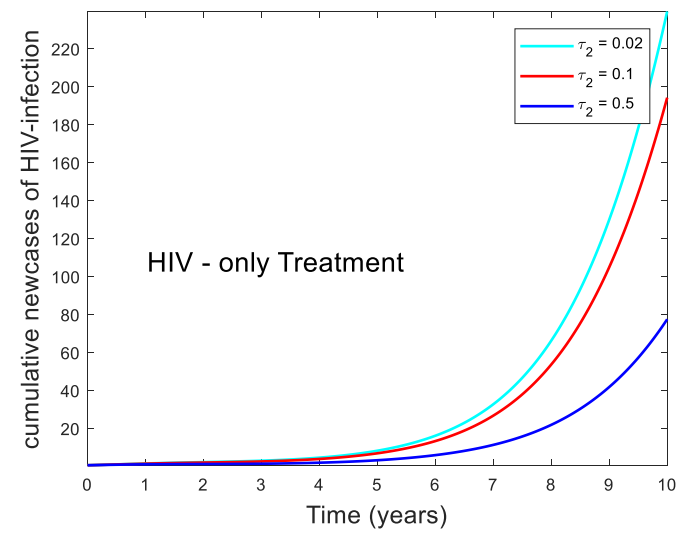

(a)

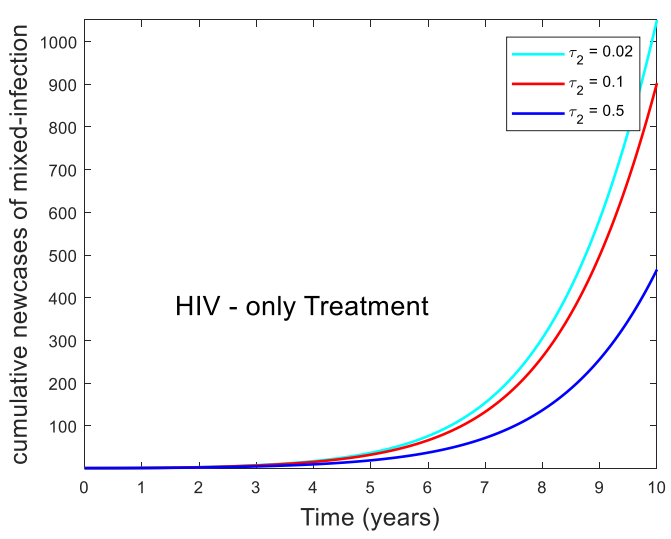

(b)

Fig. 5. The above figures (simulation of the model (6)) illustrate the cumulative number of new cases of HIV and the mixed infection showing the effect of HIV-only treatment $\left(\tau_{2}\right)$ to individuals transited with HIV-only and mixed infection. Parameter values are obtained from Table 2 with $\mu_{H}=0.01, \beta_{M}=2.6, \tau=0, \tau_{1}=$ $0, b_{M}=1$ and different values of $\tau_{2}$.

\section{Malaria-only Treatment Strategy}

Here individuals in the $I_{M}$ and $I_{H M}$ class are treated at a rate $\tau_{1}$. Here $\tau_{2}=0$ and $\tau=0$. Fig.6a and Fig. $6 \mathrm{~b}$ show that cumulative number of new cases of malaria-infection and mixed-infection have reduced remarkably. It is notable that the number of malaria cases prevented (Fig.6a) under the malariaonly treatment strategy is greater than the number of HIV cases prevented under the HIV-only treatment strategy (Fig.5a). The result follows the similar trends for higher treatment rate (Fig.6.).

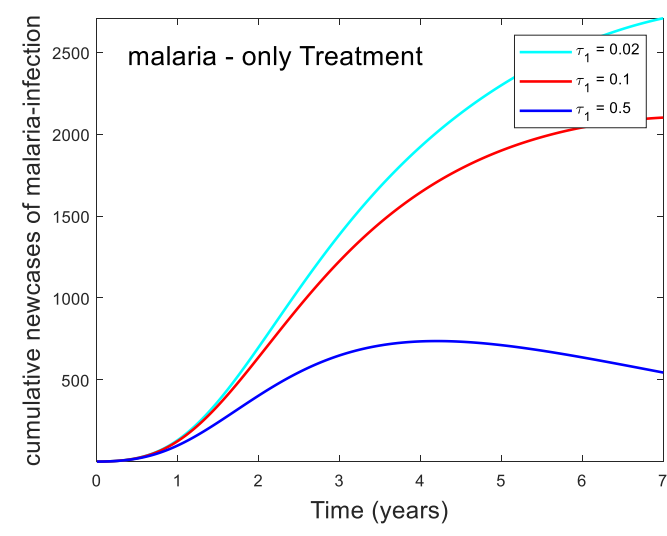

(a)

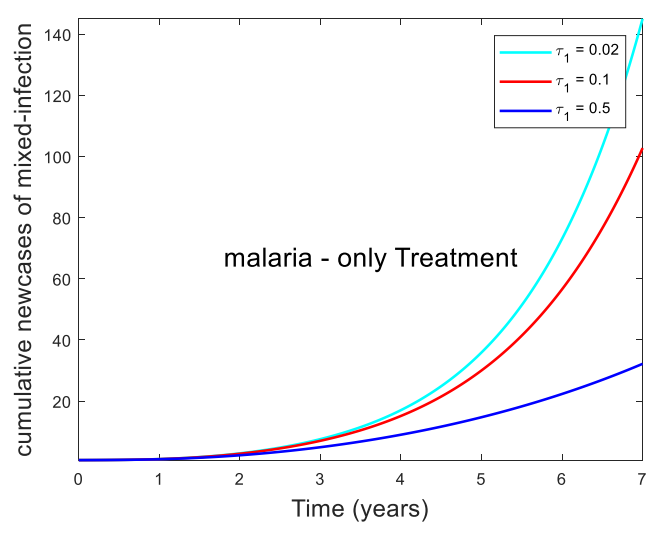

(b)

Fig. 6. The above figures (simulation of the model (6)) illustrate the cumulative number of new cases of malaria and the mixed infection showing the effect of malaria-only treatment $\left(\tau_{1}\right)$ to individuals transited with malaria-only and mixed infection. Parameter values are obtained from Table 2 with $\mu_{H}=0.01, \beta_{M}=2.6$, $\tau=0, \tau_{2}=0, b_{M}=1$ and different values of $\tau_{1}$. 


\section{Mixed Treatment strategy}

Here individuals in the $I_{H}, I_{M}$ and $I_{H M}$ class are treated at a rate $\tau$. In this case $\tau_{1}=\tau_{2}=0$. The results obtained for mixed treatment using $\tau=0.02$, depicted in Figs. 7a-7c show that this treatment strategy reduces more cumulative new cases of mixed infection than HIV and malaria infection. However, this strategy saves less number of new cases than the other two treatment strategies. The result follows the similar trends for higher treatment rate (Fig. 7.).

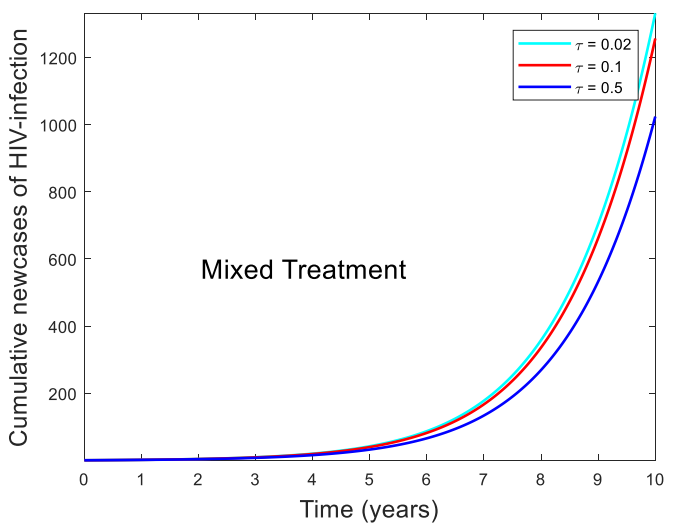

(a)

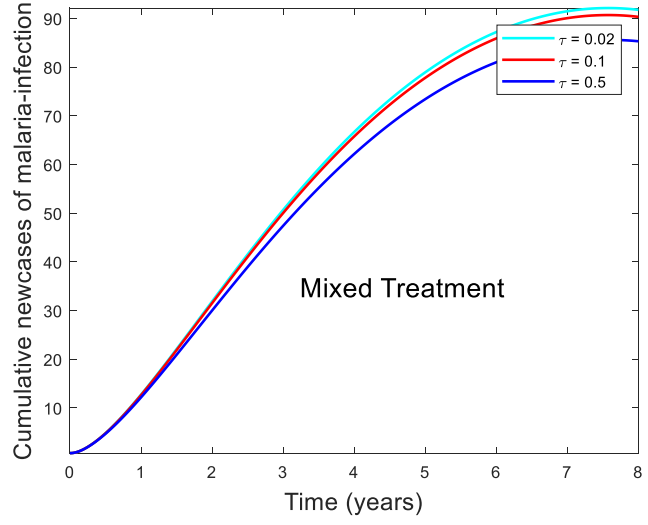

(b)

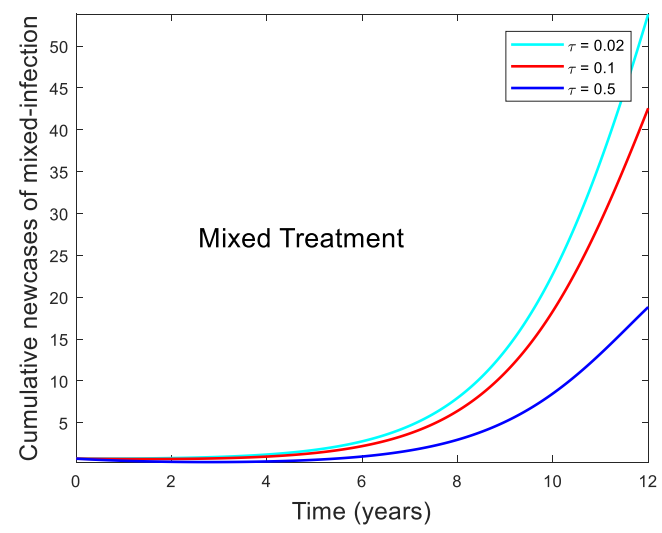

(c)

Fig. 7. The above figures (simulation of the model (6)) illustrate the cumulative number of new cases of HIV malaria and the mixed infection showing the effect of mixed treatment $(\tau)$ to individuals transited with HIVonly, malaria-only and mixed infection. Parameter values are obtained from Table 2 with $\mu_{H}=0.01, \beta_{M}=$ $1, \tau_{1}=0, \tau_{2}=0, b_{M}=1$ and different values of $\tau$.

\section{Conclusions}

The main theoretical results obtained are as follows:

1. The HIV-only model has a globally-asymptotically stable disease-free equilibrium when the reproduction number $\left(\mathcal{R}_{H}\right)$ is less than one and unstable when $\mathcal{R}_{H}$ is greater than one; 
2. The HIV-only model has a unique endemic equilibrium whenever $\mathcal{R}_{H}$ is greater than one.

3. The malaria-only model undergoes the phenomenon of backward bifurcation, where the stable disease-free equilibrium co-exists with a stable endemic equilibrium when the corresponding reproduction number $\left(\mathcal{R}_{M}\right)$ is less than one.

Using numerical simulations the following results are found:

1. The full HIV-malaria model has a locally-asymptotically stable disease-free equilibrium when $\mathcal{R}_{H M}$ is less than one, and unstable if $\mathcal{R}_{H M}$ is greater than one. It also undergoes the phenomenon of backward bifurcation when we impose some conditions.

2. HIV-only and malaria-only treatment strategy resist more cumulative new cases of the respective diseases than the mixed infection;

3. The malaria-only treatment strategy saves more cumulative new cases than the HIV-only treatment strategy for the mixed infection;

4. The mixed treatment strategy is less effective than the other two treatment strategies as it saves the least number of cumulative new cases of HIV, malaria and the mixed infection.

\section{REFERENCES}

[1] Abu-Raddad, L. J., Patnaik, P. and Kublin, J. G. "Dual infection with HIV and malaria fuels the spread of both diseases in sub-Saharan Africa." Science 314, no. 5805 (2006): 1603-1606.

[2] Baird, J. K. "Resurgent malaria at the millennium.” Drugs 59, no. 4 (2000): 719-743.

[3] Barley, K., Murillo, D., Roudenko, S., Tameru, A. M. and Tatum, S. "A mathematical model of HIV and malaria coinfection in sub-saharan Africa." Journal of AIDS and Clinical Research 3, no. 7 (2012): 1-7.

[4] Biswas M. H. A. "On the Evolution of AIDS/HIV Treatment: An Optimal Control Approach", Current HIV Research, 12 (1):1-12, 2014.

[5] Carr, J. “Application of center manifold.” Springer-Verlag, New-York (1981).

[6] Castillo-Chavez, C. and Song, B. "Dynamical models of tuberculosis and their applications." Mathematical biosciences and engineering 1, no. 2 (2004): 361-404.

[7] Center for Disease Control and Prevention (CDC)(2017)(Accessed May, 2017).

[8] Gumel, A. B. and Song, B. "Existence of multiple-stable equilibria for a multi-drug-resistant model of Mycobacterium tuberculosis." Mathematical biosciences and engineering: MBE 5, no. 3 (2008): 437-455.

[9] Hethcote, H. W. "The mathematics of infectious diseases." SIAM review 42, no. 4 (2000): 599-653.

[10] Jegede F. E., Oyeyi T. I., Abdulrahman S. A., Mbah H. A., Badru T., Agbakwuru C., et al. (2017) "Effect of HIV and malaria parasites co-infection on immune-hematological profiles among patients attending antiretroviral treatment (ART) clinic in Infectious Disease Hospital Kano, Nigeria." PLoS ONE12, no. 3.

[11] LaSalle, J. P. "The stability of dynamical systems, Regional Conference Series in Applied Mathematics, SIAM, Philadelphia, 1976." Khalid Hattaf Department of Mathematics and Computer Science, Faculty of Sciences Ben M'sik, Hassan II University, PO Box 7955 (2012).

[12] Li, X. Z., Gao, S. S. and Martcheva, M. "Modeling and control of malaria when mosquitoes are used as vaccinators." Mathematical Population Studies 22, no. 3 (2015): 145-171.

[13] Mukandavire, Z., Gumel, A. B., Garira, W. andTchuenche, J. M. "Mathematical analysis of a model for HIV-Malaria co-infection." (2009). 
[14] Nyabadza, F., Bekele, B. T., Rúa, M. A., Malonza, D. M., Chiduku, N. andKgosimore, M. "The implications of HIV treatment on the HIV-Malaria coinfection dynamics: a modeling perspective." BioMed research international 2015 (2015).

[15] Sahani S. K., Islam A. and Biswas M. H. A. "Mathematical Modeling Applied to Understand the HostPathogen Interaction of HIV Infection in Bangladesh", Surveys in Mathematics and its Applications, 12, (2017) 165-178.

[16] Sharomi, O., Podder, C. N., Gumel, A. B., Elbasha, E. H., and Watmough, J. "Role of incidence function in vaccine-induced backward bifurcation in some HIV models." Mathematical Biosciences 210, no. 2 (2007): 436-463.

[17] Sharomi, O., Podder, C. N., Gumel, A. B., and Song, B. "Mathematical analysis of the transmission dynamics of HIV/TB confection in the presence of treatment." Mathematical Biosciences and Engineering 5, no. 1 (2008): 145.

[18] Ukaga, C. N. "HIV-malaria coinfection: Anti-malaria therapy in people living with HIV/AIDS in Owerri, Nigeria." International Society for Infectious Diseases 21, Supplement 1, (2014) Page 121.

[19] United Nations Department of Economic and Social Affairs/Population Division (2004). The impact of AIDS. United Nations, New York.

[20] Van den Driessche, P., and Watmough, J. "Reproduction numbers and sub-threshold endemic equilibria for compartmental models of disease transmission." Mathematical biosciences 180, no. 1 (2002): 29-48.

[21] Winiarti, D., Mudigdo, A. and Murti, B. "Determinants of Recurrence and Death in HIV-Malaria CoInfection Patients in Jayapura, Papua, Indonesia.” Journal of Epidemiology and Public Health 4, no. 3 (2019).

[22] World Bank, (1997). Confronting AIDS: public priorities in a global epidemic. Oxford university press.

[23] World Health Organization (2017) (Accessed May, 2017). 